Ans dem Königl. Institat für Geburtshälfe und Gynäkologie an der Universităt Rom. Direktor: Prof. E. Pasquali.

\title{
Ueber das Vorhandensein von Protozoën bei der Endometritis chronica glandularis.
}

\author{
Von \\ Dr. Tullio Rossi Doria ${ }^{1}$,
}

Assistenten am Institut.

(Mit 6 Abbildungen auf Tafel I.)

Unter den 21 Endometritisfällen, die während des vergangenen Studienjahres am Kgl. Institut für Geburtshülfe und Gynäkologie in Rom mittels Auskratzung behandelt und von mir vom Gesichtspunkt der pathologischen Anatomie und der Aetiologie genauer untersucht wurden, zogen zwoi meine Aufmerksamkeit auf sich, nicht sowohl wegen ihres histo-pathologischen Befundes, welcher in einer Endometritis glandularis hypertrophica bestand, als wegen des Vorhandenseins von Körpern, die Protozoën (Amöben) durchaus ähnlich waren und sich sowohl im Lumen der cystös hier und da erweiterten Drüsen, als auch im Innern der Bekleidungsepithelzellen zeigten.

Mein erster Gedanke war, die im Innern der Drüsenschläuche enthaltenen Körperchen wären abgeblätterte und durch Anschwellung im Innern der serös-mucinartigen, die Drüsen füllenden Flüssigkeit, veränderte Epithelzellen und die in den Zellen des Epithels eingeschlossenen Körperchen wären nichts Anderes als eingeschlossene

1) Aus dem italienischen Manuscript übersetzt von Herrn Dr. phil. Loevinson in Rom. 

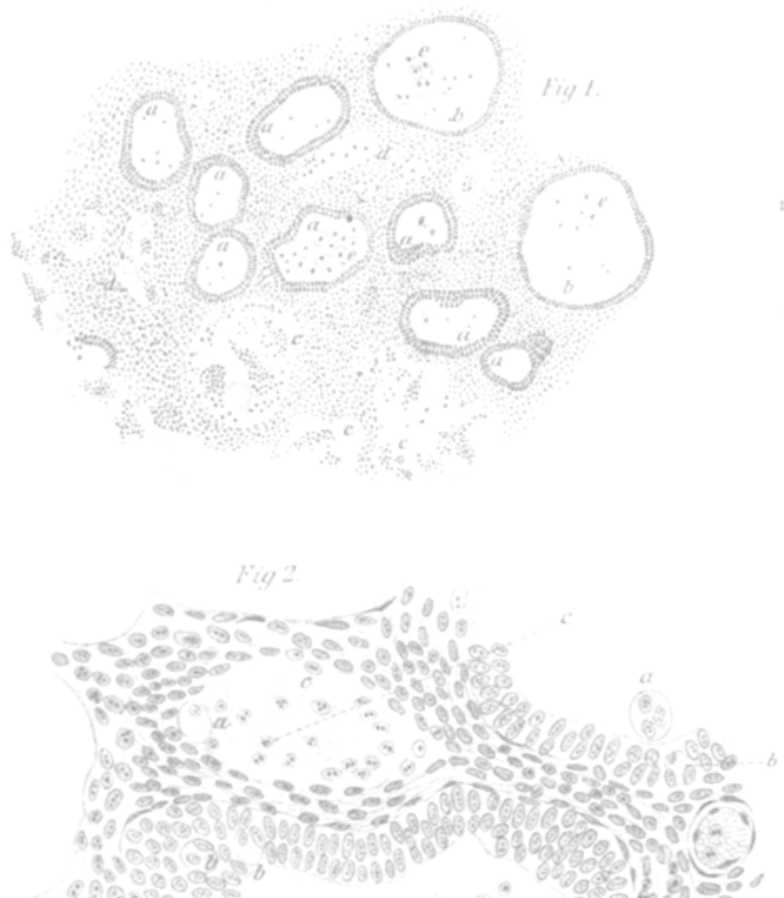
$\infty, 8,0,0000$

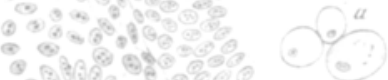
(3) $3,3,3$,

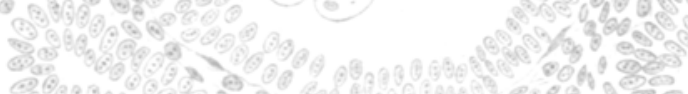

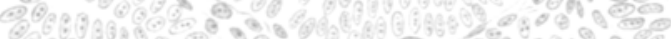

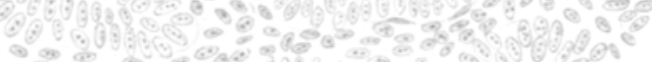

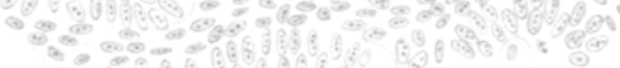
3
3

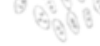

$$
\text { Q) }
$$

Fig.t

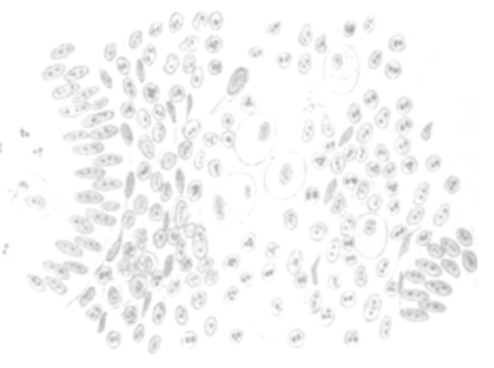

Fig. 5
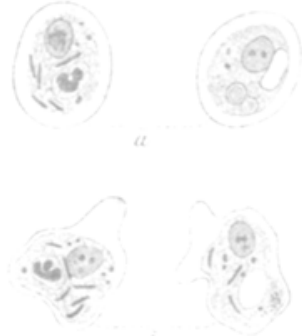
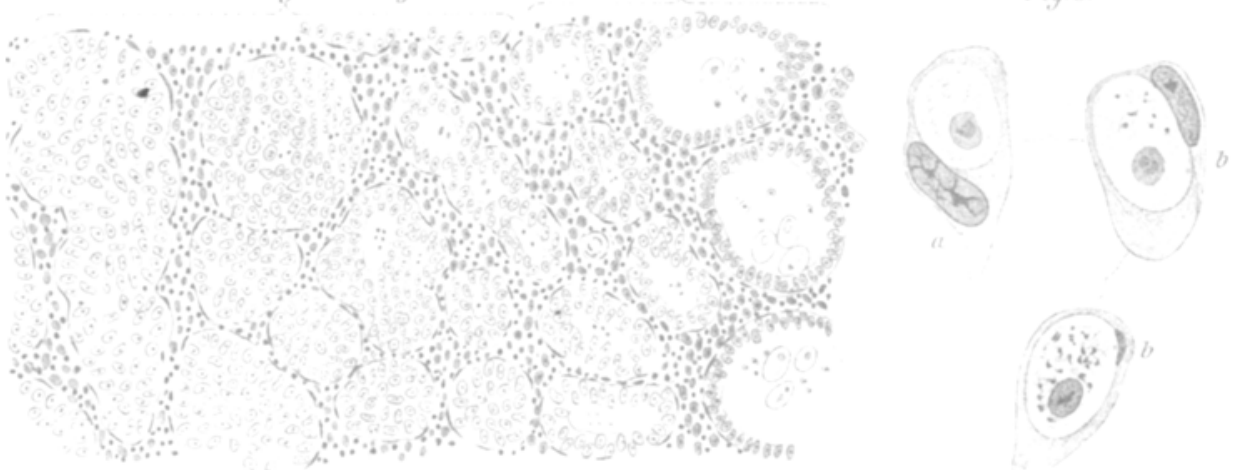

ivis 
Zellenelemente (Zelleneinschliessung), wie sie von vielen Autoren bei manchen Neubildungen und speciell beim Carcinom beschrieben worden sind.

Jedoch bei genauerer Untersuchung der Präparate dieser beiden Fälle und bei Untersuchung vieler anderer Präparate von Endometritis, die von mir und mehreren meiner Kollegen histologisch geprüft wurden, sah ich, dass es nicht möglich war, die genannten Körperchen mit angeschwollenen oder in anderer Weise veränderten und entarteten Epithelzellen zu verwechseln.

Denn sie waren grösser als die Epithelzellen, 5-8 Mal so gross als ein rothes Blutkörperchen (die im Innern der Gewebe befindlichen waren etwas kleiner als die freien), von meist sphärischer, manchmal leicht verlängerter (ovoïder, ellipsoïder) Form. Sie enthielten einen viel weniger intensiv färbbaren und kleineren Kern als der der Epithelzellen ist; er war ferner blasenartig, mit vereinzelten und nicht constanten Kernchen. Das Protoplasma war meistens vacuolisirt und zerstreut, mit Ausnahme eines hyalinen, peripherischen Randes von Granulationen und fremden Körpern. Unter diesen bemerkte ich Bakterien, rothe Blutkörperchen und Leukocyten. (Siehe Abbild. 4.)

Es fehlte keines der Merkmale, welche als characteristisch für die Amöben beschrieben worden sind. Trotzdem musste ich, da es weder chemische noch physische Mittel giebt, um in sicherer Weise die todten Amöben von den anderen Zellen, welche die Gewebe und speciell pathologische zusammensetzen, zu unterscheiden, die Gelegenheit abwarten, um im lebenden Zustand diejenigen Körperchen zu untersuchen, in denen ich bisher Amöben nur vermuthen, aber nicht sicher erkennen konnte, und um ihre characteristischen Bewegungen zu entdecken.

Der Zufall war mir günstig; denn einige Zeit darauf hatte ich mit einem dritten Fall von Endometritis glandularis zu thun, in welchem ich durch Zerfetzung aus den abgekratzten Wucherungen Präparate herstellte und diese im hängenden Tropfen einer physiologischen Lösung von Kochsalz untersuchte. In demselben vermochte ich zahlreiche, von nicht sehr schnellen, aber ziemlich deutlichen Bewegungen belebte Amöben zu erkennen. Dank der hohen Sommertemperatur war keine Erwärmung des Gläschens nothwendig, um die Bewegungen des Protoplasmas zu bemerken. Eine Vergrösserung von 400 Durchmessern war mehr als genügend zur Aufsuchung und Verfolgung der Amöben. 
Da sonach betreffs des Vorhandenseins dieser Protozoën in den von mir untersuchten Fällen von Endometritis glandularis kein Zweifel geblieben war, schien es angemessen, die Aufmerksamkeit auf diese Thatsache zu lenken; um so mehr als sie schon früher von Histo-Pathologen beobachtet worden ist.

In der Literatur über Gebärmutterkrankheiten findet sich, so viel ich weiss, das Vorhandensein von Amöben im Endometrium nirgends besprochen. Immerhin jedoch sind Körperchen, denen ähnlich, die ich gesehen habe, nicht nur in der Schleimhaut der Gebärmutter, sondern auch in der Muttertrompete bemerkt worden. Sie wurden für Deciduaelemente gehalten, und möglicherweise handelte es sich in vielen Fällen, soviel aus der Beschreibung der mikroskopischen Präparate hervorgeht, wirklich um Deciduazellen, um eine pathologische Decidua, die mit derjenigen der Schwangerschaft nicht verwechselt werden darf. Jedoch in drei Fällen von Gebärmutterkrebs, welche von Chiari') beschrieben worden sind, finden sich Elemente für deciduale angesehen, welche, wie es nach der Beschreibung scheint, den von mir gefundenen Amöben sehr ähnlich sind. In diesen drei Fällen handelte es sich um Tumoren, welche aus der Schleimhaut hervorgingen und einen bösartigen Verlauf hatten.

Später hat Ferrari²) dieselben, den decidualen ähnliche Elemente in der Schleimhaut der Trompete bei einem Falle von Salpingitis in einer von der Schwangerschaft entfernten Epoche gefunden. Er sah diese Zellen in den Zwischenräumen zwischen den einzelnen Falten der Schleimhaut. Sie waren manchmal in Gruppen von 5 oder 6 vereinigt. Der Kern dieser grossen Elemente färbte sich gut, aber nicht intensiv. Das Protoplasma zeigte Vacuolen und verschiedene Granulationen; sie waren von einer Grösse, die zwischen 10,00 $\mu$ und $25 \mu$ variirte. Die Abbildungen, welche Ferrari davon giebt, sind derartig, dass man glauben kann, eins von meinen Präparaten vor Augen zu haben. Er ist hinsichtlich der Erklärung dieser pseudodecidualen Elemente, wie er sie nennt, unsicher und bemerkt ganz richtig, sie müssen einen von dem der wirklichen Deciduazellen ganz verschiedenen Ursprung haben.

1) Ueber 3 Fälle von primärem Carcinom im Fundus und Corpus des Uterus. Oestr. Med. Jahrbücher 1877.

1) Cellule simili alle deciduali sulla mucosa tubarica. Annali di Ostetricia e Ginecologia. Anno XIV, p. 567. 
Es möge nun die Beschreibung der einzelnen Fälle folgen.

I. Fall. S. M., 47 Jahre alt, verheirathet. Hat zuletzt vor 6 Jahren geboren und niemals an irgend einer Beschwerde in der Genitalgegend gelitten; Menstruation stets regelmässig, nie von Schmerz begleitet. Seit ungefähr zwei Monaten leidet die Kranke an bald stärkerem, bald geringerem Blutverlust, magert $a b$ und ist stark anämisch. Sie klagt ausserdem über Lendenschmerzen und eine gewisse Beschwerde beim Urinlassen.

Bei der Untersuchung findet sich der Uterus beweglich, von normaler Grösse und Form und in physiologischer Lage. Die Sonde dringt unter einem gewissen, jedoch nicht sehr heftigen Schmerz $71 / 2 \mathrm{~cm}$ tief ein. Nach ihrer Entfernung zeigt sich ein bedentender Ausfluss von mit dünnem gelblichem Schleim gemischtem Blute. Prüft man mit der Sonde die Wände der Gebärmutterhöhle, so nimmt man ziemlich bedeutende Unebenheiten oder pilzähnliche Ansätze wahr.

Therapie. Ausschabung der Gebärmutterhöhle.

Erfolg. Vollkommene Heilung. Die Mutterblutungen hören auf, und die Frau verlässt nach einer reconstituirenden Kur die Klinik im besten Gesundbeitszustand.

Pathologische Anatomie. Die Untersuchung der entfernten Schleimhaut ergiebt eine ausserordentliche Entwickelung der Gebärmutterdrüsen, welche nicht blos an Zahl vermehrt, sondern auch so erweitert sind, dass sie in einigen Punkten wahre Cystenhöhlen bilden. Das Bekleidungsepithel zeigt bei einigen Drüsen, wie in normalem $\mathrm{Zu}$ stande, eine einzige Reihe von Zellen, bei anderen jedoch ist die Epithelwucherung so bedentend, dass sie zur Bildung einer doppelten Reihe dicht zusammengedrängter Zellen gefübrt hat. Diese bewahren ihre Cylinderform ziemlich gut dort, wo die Erweiterung der Drüse nicht zu gross ist; da hingegen, wo die Drüse sich cystisch erweitert, wird das Epithel fast kubisch.

Im Innern der Drüsen sieht man dicke, protoplasmatische, rundliche, meist sphärische, bisweilen etwas verlängerte Massen, die mit einem nicht sehr färbbaren, häufig vacuolisirten Kern von der 5-8maligen Grösse eines rothen Körperchens versehen sind. Untersucht man sie genau, so lassen sie, nicht alle jedoch und auch nicht mit gleicher Leichtigkeit, einen hyalinen peripherischen Rand (Ectosarca) und einen centralen Theil (Endosarca) erkennen, der mit Körnchen, mit Vacuolen und mit fremden Körpern wie rothen Blutzellen und Leukocyten bestreut ist.

Aehnliche, obschon etwas kleinere protoplasmatische Massen finden sich ebenfalls innerhalb der Schleimgewebe sowie innerhalb der Epithelzellen selbst, welche dadurch in ihrer Form und Grösse verändert werden. Diese erscheinen nämlich infolge des Vorhandenseins dieser Amöben bedeutend grösser, sphärisch oder beinahe sphärisch; ihr nach der Peripherie zu verschobener Kern ist manchmal erhalten; an anderen Stellen - jedoch verschwindet er und geht zu Grunde, ein anderer blasserer und kleinerer Kern erscheint in der so umgestalteten Zelle, und die Körnchen und Vacuolen der Amöben lassen eine Entartung eben dieser Zelle vermuthen. Dieser Anñahme gab ich mich hin, als ich die Präparate zum ersteu Male beobachtete, bevor ich mich überzeugte, dass es sich um parasitäre, in den Zellkörper eingedrungene Amöben handelte.

Das mit zahlreichen Infiltrationszellen bestreute Interglandular- 
gewebe ist ebenfalls verändert mit seinen hier und da erweiterten, manchmal ungeheuer an Zahl gewachsenen Capillaren. Zahlreiche Blutergüsse finden sich in dem ganzen Gewebe und speciell an der Oberfläche verstreut. So erklären sich die reichlichen, fortdauernden Hämorrhagien, an denen die Frau litt.

Wie sehr ich auch sowohl mit der Gram'schen als auch mit anderen Färbungsmethoden nach Bakterien gesucht habe, so habe ich doch keine Bakterienart weder im Interstitialgewebe noch in den Drüsen bemerken können.

II. Fall. R. A., 43 Jahre alt, verheirathet. Hat zuletzt vor drei Jahren geboren und sich seit dieser Zeit nicht mehr gut befunden. Die Menstruation, die stets regelmässig gewesen war, wurde bald dürftig, bald reichlich, häufig schmerzhaft. Seit einiger Zeit war ein weisslicher Ausfluss aufgetreten, der schliesslich durch reichliche Metrorrhagien gebildet wurde, welche fortdauernd und erschöpfend bereits seit ungefähr 14 Tagen andauerten.

Bei der Untersuchung der Frau findet sich die Gebärmutter beweglich, in physiologischer Lage etwas vergrössert, schmerzhaft. Die Sonde dringt $8 \frac{1}{2} \mathrm{~cm}$ ein; nach ihrer Entfernung tritt Ausfluss einer serösmucinartigen, blutigen Flüssigkeit ein. Ein solcher von Blut allein, und zwar reichlich, erfolgt, wenn man die schwammigen Massen berührt, die man deutlich innerhalb der Gebärmutterhöhle wahrnimmt. Gebärmutterhals hypertrophisch, blutüberfüllt.

Therapie. Ausschabung der Gebärmutterhöhle.

Erfolg. Befriedigend. Die Frau verlässt das Krankenhaus anscheinend vollkommen geheilt. Blutabgang und Schmerzen verschwunden, das Allgemeinbefinden sehr gut. Nach einem Monat jedoch waren von neuem ziemlich reichliche Metrorrhagien aufgetreten. Aber die Kranke liess sich nicht sehen, wurde gesucht und konnte nicht aufgefunden werden.

Pathologische Anatomie. Derselbe Befund wie im vorhergehenden Fall, nur dass sich überall zahlreiche Bakterienformen vorfanden, und ein höherer Grad von Infiltration des Interstitialgewebes vorhanden war. Einige Drüsen waren mit Eiter angefüllt; überaus zahlreiche Leukocyten lagen in den Lymphräumen und im Innern der erweiterten Capillaren. Die Amöben waren auch hier in beträchtlicher Anzahl vorhanden, sowohl innerhalb des Lumens der Drüsen als auch im Innern der Gewebe.

III. Fall. De T. V., 29 Jahre alt, Wittwe. Nullipara. Immer gesund. Menstruation regelmässig bis vor zwei Jahren. Seit dieser Zeit und namentlich seit ungefähr 14 Tagen überreichliche Metrorrhagien, welche die Frau pyhsisch und moralisch herunterbringen.

Bei der Untersuchung findet sich die Gebärmutter beweglich, in physiologischer Lage, etwas geschwollen. Die Einführung der Sonde erregt einen dumpfen Schmerz; sie dringt $7 \frac{1}{2} \mathrm{~cm}$ tief ein, und beim Herausziehen fiesst ans der Mündung Blut in grosser Quantität hervor. Beim Berühren der Gebärmutterwände bemerkt man das Vorhandensein von Schwammgewächsen. Collum hyperämisch, ein wenig aufgeschwollen; aus seiner Mündung kommen Schwammgewächse hervor.

Therapie. Ausschabung der Gebärmutterhöhle und des Gebärmutterhalses.

Erfolg. Sehr gut. Die Frau genas vollkommen und verliess das 
Krankenhaus in bestem Zustand. In der Folge hat sie regelmässig ihre Menstruationen gehabt und über nichts geklagt.

Pathologische Anatomie. Befund dem vorhergehenden gleich, nur dass die Veränderungen der Gewebe weniger vorgeschritten erschienen. Um mich in sicherer und definitiver Weise von der Natur der bereits beschriebenen Körperchen zu überzeugen, trug ich Sorge, ihren Tod weder abzuwarten noch zu befördern, sondern die Schleimhaut im frischen Zustand zu untersuchen, und die Bewegungen der lebenden Amöben zu erforschen. Die entfernten Gewebe wurden daher sofort im hängenden Tropfen in physiologischer Kochsalzlösung bei atmosphärischer Temperatur $\left(34-36^{\circ}\right)$ untersucht.

Die Amöben waren ziemlich zahlreich, zeigten noch deutlicher als in den gefärbten Präparaten das Ectosarca von dem Endosarca unterschieden und bewegten sich langsam aber ganz deutlich. Bei allen Bewegungen war das, was heraustrat, das Ectosarca. Es verdickte sich bald auf der einen, bald auf der anderen Seite und verlängerte sich in Pseudopodien, während man beim Endosarca eine passive Bewegung der Vacuolen und der darin enthaltenen Körnchen bemerkte. Ich habe unter dem Mikroskop ziemlich deutlich auch die Einverleibung fremder Substanzen und die Ausstossung bereits vom Parasiten ausgenutzter Producte sehen können. Kein Zweifel ist mir daher geblieben, als ob es sich nicht um wirkliche Amöben handele. Zehn bis zwölf Stunden später waren die Bewegungen der Amöben viel langsamer, und sie erschienen alsdann fast alle rundlich, in ihrer Ruheform durchaus denjenigen Amöben gleichartig, welche man bei den gefärbten Präparaten sieht.

Trotz allen Suchens gelang es mir bei keiner der Amöben einen Beginn von Sporulation zu finden; es ist daher nicht unwahrscheinlich, dass es sich um Formen handelt, welche sich durch Spaltung vervielfältigen.

Fassen wir das Gesagte kurz zusammen, so fanden sich in allen drei Fällen von Endometritis glandularis cystica sowohl innerhalb der Drüsen in freiem Zustand, als innerhalb der Zellen Amöben; in allen drei Fällen war der klinische Charakter der Hämorrhagie dem gleich, wie er sich fast constant bei den durch Protozoën hervorgerufenen Affectionen findet (Dysenterio etc.), in allen drei Fällen fand eine Hyperplasie der Epithelien statt, welche die Zellenelemente sind, auf welche vorwiegend die Reiz erregende Action der Protozoën ausgeübt wird; in allen drei Fällen (mit Ausnahme eines, über den einiger Zweifel aufkommen kann) hatte die Ausschabung der Schleimhaut vortreffliche Wirkung und erzielte völlige Heilungen.

Gegenüber diesen Thatsachen tauchen von selbst mehrere und vor allen folgende Fragen auf: Sind die vorgefundenen Protozoën die Ursache der Endometritis und der speciellen Form derselben? Sind sie die einzige Ursache derselben?

Die Antwort darauf ist keine leichte. Nur in einem der zu 
meiner Beobachtung gelangten Fälle waren die Amöben allein, d. h. wenigstens dem Anscheine nach nicht von Bakterien begleitet; in den beiden anderen Fällen waren solche sowohl innerhalb des Lumens der Drüsen als auf dom Rande der ulcerösen Gewebe und in den Lymphräumen des Interstitialbindegewebes vorhanden, Die pathogene Kraft dieser Bakterien habe ich nicht experimentell feststellen können; und andererseits sind unsere Kenntnisse über die Action der verschiedenen Bakterien, welchen man so häufig im Uterus der von chronischen Schleimhautentzündungen ergriffenen Frauen begegnet, derart dürftig, dass das Experimentiren ihrer pathogenen Kraft auf die Thiere die Frage vielleicht nur wenig gefördert hätte.

Andererseits waren die Amöben in allen drei Fällen vorhanden und zwar in beträchtlicher Zahl, hatten die Gewebe angegriffen und beschränkten sich nicht darauf, innerhalb der Drüsen-Lumina za bleiben, sondern drangen auch in die Epithelzellen selbst ein.

Alles wohl erwogen, halte ich für die richtigste oder wenigstens verständigste Ansicht, dass man, so lange nicht neve Untersuchungen grösseres Licht über die Frage verbreitet haben, für wabrscheinlich annimmt, es handle sich in diesen, wie in anderen ähnlichen Fällen um eine gemischte Wirkung, die von den Bakterien und den Amöben zusammen ausgeübt wird. Die Amöben können vielleicht dem Eindringen der Bakterien den Weg öffnen, ebenso wie diese auf das Wachsthum ersterer einen günstigen Einfluss ausüben mögen; oder auch es arbeitet ein jedes dieser beiden Agentien für sich besonders, ohne die Hülfe des andern nöthig zu haben und womöglich ohne dessen Einfluss zu verspüren; sind sie darauf in das Innere der Gewebe eingedrungen, so bestimmen sie dort diejenigen Veränderungen, aus denen die verschiedenen klinischen Bilder, die mannigfaltigen anatomisch-pathologischen Formen hervorgehen.

Es ist jedoch nicht unwahrscheinlich, dass die Amöben die Bedentung eines ätiologischen Factors erster Ordnung haben können, wie andere, allgemein in der wissenschaftlichen Welt angenommene Thatsachen beweisen. Denn von der Malariainfection ist es jetat zweifellos erwiesen, dass sie von Amöben hervorgebracht wird (Colli und Marchiafava, Golgi, Grassi etc.).

Bei der Dysenterie begegnet man Amöben, wie von Lösch an sehr viele Forscher gesehen haben. Kruse und Pasquale an der zoologischen Station in Neapel haben auf ihrer wissenschaft- 
lichen Expedition nach Aegypten zum Studium der Dysenterie and des hepatischen Abscesses bei den Katzen durch Inoculirung der Amöben in den Mastdarm eine der Dysenterie ähnliche Form gefunden, mit Läsionen der Schleimhaut, welche den von mir im Endometrium gefundenen ähnlich und speciell in den Drüsen localisirt waren, die mit den genannten Parasiten angefüllt waren. Man achte darauf, dass die Reproduction dieser Form auch durch Inoculirung von. Eiter eines hepatischen Abscesses, der nur Amöben enthielt, ohne irgend eine Spur von Bakterien, wie man mit Hülfe der Culturen sehen konnte, vorgenommen wurde.

In gleicher Weise zweifelt man seit einiger Zeit nicht mehr an einer Infection, welche man häufig in der Leber des Kaninchens antrifft und welche Coccidiosis genannt wird, weil sie vom Coccidium oviforme hervorgebracht wird. Dasselbe ist keine Amöbe, aber ein den Amöben nahe verwandtes Protozoon. Sein Vorhandensein in den Gallenkanälen und sein Eindringen in die Zellen der Epithelbekleidung erzeugt eine Erweiterung der Kanäle sowie eine Proliferation des Epithels, und kann sogar, wiewohl selten, wirkliche Tumoren vom Typus der Cystoadenome hervorbringen.

Da haben wir also eine Proliferation von Epithelelementen, die sicher dem Einfluss einer von Protozoën verursachten Infection zuzuschreiben ist.

Ich will hier nicht das Molluscum contagiosum, noch Paget's Krankheit noch die anderen verschiedenartigen Affectionen der verschiedenen Epithele, welche den Protozoën zugeschrieben werden, citiren, und noch viel weniger will ich vom Carcinom und seinem parasitären Ursprung, nach der Meinung vieler Forscher, reden.

Ich kann jedoch nicht umhin zu bemerken, wie diese Formen Fon Endometritis glandularis sich, man kann sagen, unmerklich, stufenweise fortsetzen, mit jenen Formen von Adenom der Gebärmutterschleimhaut, wolche so häufig einen bösartigen Verlauf nehmen und schliesslich den Namen wirklicher Krebse verdienen. Küstner ${ }^{1}$ ) drückt sich in dieser Beziehung folgendermassen aus: "Wir sind bis zu einem gewissen Grade berechtigt, die glanduläre Form der Endometritis hyperplastica als diffuses Adenom der Uterusschleimhaut za bezeichnen."

1) 0. Küstner, Grundzüge der Gynäkologie. Jena 1893. 
Und vor ihm Wyder ${ }^{1}$ : „Wenn man sich die Aufgabe stellt, die Endometritis glandularis, das Adenoma benignum und malignum genau zu definiren and zwischen diesen Formen eine scharfe Grenze zu ziehen, so stellt sich heraus, dass dies geradezu ein Ding der Unmöglichkeit ist, indem ohne $Z$ weifel zwischen den genannten Affectionen mannigfache Uebergänge existiren."

In einigen der von mir beobachteten Fälle habe ich mich wirklich in Verlegenheit gefunden, zu entscheiden, ob es sich um ein Adenom oder eine Endometritis glandularis handle. In dem einen wio in dem anderen Falle ist gesteigerte Epithelproliferation vorhanden; und ob diese im weiteren Verlauf zu einem Krebse führen wird, indem sie eine unmöglich vorauszusehende Bösartigkeit annimmt, oder ob sie sich in den Grenzen einer Gutartigkeit, die man nicht erhoffen darf, halten wird, kann, nach meiner Ansicht Niemand entscheiden, was auch immer Döderlein ${ }^{2}$ ) in Zweifel's Vorlesungen darüber sagen mag.

Diese Aehnlichkeit der beiden Processe, welche nach den anerkanntesten Theorien von durchaus verschiedener Natur wären, würde nun eine ganz befriedigende Erklärung finden, wenn man statt dessen beweisen könnte, dass die Ursache, welche die Endometritis glandularis, ebenso auch das Adenom, auch den Krebs der Gebärmatter hervorbringt.

Und dies würde durchaus nicht wunderbar erscheinen, wenn man bedenkt, dass den meisten Uteruskrebsen ein Reizzustand der Schleimhaut, d. h. Endometritis-Symptome vorangehen; wenn man ferner bedenkt, dass die Gebärmutterschleimhaut in naher Verbindung zu der an Infectionselementen reichen äusseren Umgebung steht, dass der Krebs des Gebärmutterhalses, der mit dieser äusseren Umgebung in unmittelbarer Verbindung steht, häufiger als der des Gebärmutterkörpers ist und ihm oft vorangeht, dass schliesslich bei den nicht sehr verbreiteten, aber doch klinisch diagnosticirbaren Gebärmutterkrebsen in dem vom Tumor nicht betroffnen Theil sich fast constant eine Endometritis glandularis hyperplastica findet, welche vielleicht ein weniger fortgeschrittenes Stadium des Processes, der sich anderswo schneller und energischer entwickelt, darstellt.

1) Th. Wyder, Tafeln für den gynäkologischen Unterricht. Text p. 27.

2) P. Zweifel, Vorlesungen über klinische Gynäkologie. Berlin 1892, S. 418 . 
Was die Prognose und die Therapie betrifft, so sind, wenn man diesen Ursprung für einige der Endometriten annimmt, die daraus zu ziehenden Consequenzen ganz klar.

Die Prognose verschlimmertsich, insofern die Endometritis glandularis nicht nur "ein prädisponirendes Moment für maligne Wucherung" ist, wie Martin ${ }^{1}$ ) scharfsinnig bemerkt, sondern auch eine maligne Neubildung in potentia, geneigt früher oder später in actum überzugehen.

Hinsichtlich der Therapie erscheint die schleunige, vollkommene, sorgfältige, tiefe, von Cauterisation gefolgte Ausschabung als die beste Hülfe. Doch kann man auch ohne diese gute Resultate erhalten, wio in unseren drei Fällen und z. B. in dem von Döderlein ${ }^{2}$ ) beschriebenen, dem unsrigen klinisch sehr ähnlichen Falle.) A. $\operatorname{Martin}^{3}$ ) versichert: „Bei polypösen Formen (die, wie wir gesehen haben, der Endometritis glandularis so ähnlich sind) genügt oft für den ersten Augenblick die einfache Abkratzung mit nachfolgender Aetzung". Vor ihm hatte Schröder ${ }^{4}$ ) gesagt, dass er durch blosse Auskratzung definitive Heilungen erzielt hatte.

Da nämlich durch die Ausschabung die ganze Schleimhant beseitigt wird, so werden auch die Bewohner dieser fortgeschafft und ihre Action wird somit gehindert; und wenn die Rückfälle bei diesen Processen so häufig sind, warum sollte man dies nicht der Permanenz einiger Parasiten anf irgend einer, an ihrer Stelle belassenen Schleimhautinsel, welche sie später verlassen, um die regenerirte Schleimhaut anzugreifen, zuschreiben können?

Bei der specifischen Wirkung, welche die Chininsalze auf die Amöben haben, würde es nicht unangebracht erscheinen (was ich bisher noch nicht vermocht habe, aber bei Gelegenheit zu thun beabsichtige), die Anwendung der intrauterinen Injectionen von Lösungen mit genannten Salzen in verschiedenen Proportionen vor oder besser nach der Ausschabung zu versuchen.

1) A. Martir, Pathologie und Therapie der Frauenkrankheiten. Bd. II, S. 212.

2) Zweifel, loc. cit. S. 419.

3) A. Martin, loc. cit. S. 295.

4) Schröder, Handbuch Aufl. 7, S. 280. 


\section{Erklärung der Abbildung auf Tafel $\mathrm{I}$.}

(Die Figuren sind mit Hülfe der Abbé'schen Lichtkammer gezeichnet.)

Fig. 1. - Fall II. Endometritis glandularis cystica. Schnitt auf die GebärmutterSchleimhaut:

a Drüsen mit oylindrischem Epithel.

b Drüsen mit cubischem Epithel.

c Interstitial-Hämorrhagien.

d Erweiterte Capillaren mit zahlreichen Leukocyten.

e Protozoën. -- Vergr. 1:50.

Fig. 2. - Fall I. Endometritis glandularis cystica.

a Freie Protozoën.

b Endocelluläre Protozoën.

c Interstitial-Hämorrhagien. - Vergr. 1:200.

Fig. 3. - Fall III.

a Endometritis glandularis.

b Adeno-carcinomatöse Form mit vollen Epithelschläuchen; das Interstitialgewebe ist an einigen Stellen verschwunden.

c Protozoën. - Vergr. $1: 100$.

Fig. 4. - Fall II.

Endometritis gland. cystica. Freie Protozoën innerhalb des infiltrirten und in Auflösung begriffenen Interstitial-Gewebes. - Vergr. 1: 200.

Fig. 5. Freie Protozoën.

a im Ruhezustande,

$\mathrm{b}$ in Bewegung.

In ihrem Innem Granulationen, Bakterien, Leukocyten etc. $1: 400$.

Fig. 6. Protozoün innerhalb Epithelzellen.

a mit unversehrtem Kern,

b mit alterirtem Kern. - Vergr. 1:500. 